\title{
Percutaneous Transgastric-Transpancreatic Treatment of a Dissecting Splenic Artery Pseudoaneurysm due to Segmental Arterial Mediolysis
}

\author{
Pradesh Kumar $^{1}$ Jasmine Ming Er Chua ${ }^{1}$ Jared Jue Ying Yeo ${ }^{1}$ Edward Tieng Chek Choke ${ }^{2}$ \\ Pooja Sachdeva ${ }^{3}$
}
${ }^{1}$ Department of Vascular and Interventional Radiology, Singapore General Hospital, Singapore
${ }^{2}$ Department of Vascular Surgery, Sengkang General Hospital, Singapore
${ }^{3}$ Department of General Medicine, Sengkang General Hospital, Singapore

\begin{abstract}
Address for correspondence Pradesh Kumar, MBChB, MRCS, FRCR, Department of Vascular and Interventional Radiology, Singapore General Hospital, Outram Road, 169608, Singapore (e-mail: pradeshkumar@doctors.org.uk).
\end{abstract}

J Clin Interv Radiol ISVIR:2020;4:111-114

\begin{abstract} Dissecting splenic artery pseudoaneurysm due to segmental arterial mediolysis (SAM) Keywords

- splenic artery pseudoaneurysm

- thrombin

- segmental arterial mediolysis is a rare condition. We describe a case of direct percutaneous transgastric-transpancreatic thrombin injection into a dissecting splenic artery pseudoaneurysm due to SAM. The direct thrombin injection resulted in successful thrombosis of the pseudoaneurysm. At 1-month follow-up, the patient remained well with persistent thrombosis of the pseudoaneurysm.
\end{abstract}

\section{Introduction}

A splenic artery pseudoaneurysm (SAP) is a rare but potentially fatal condition. Endovascular management is usually the first-line treatment option for SAPs. However, when conventional transarterial embolization (TAE) treatment fails, a direct percutaneous option can be considered. Here, we report a case of thrombin injection through ultrasound-guided percutaneous transgastric-transpancreatic access. To our knowledge, there have been no published reports of this unique technical approach in the literature.

\section{Case History}

A 35-year-old man presented with a 1-week history of constant epigastric abdominal pain associated with nausea and vomiting. Systemic review was unremarkable. His past medical history included gastritis, generalized anxiety disorder, and unexplained infertility. On physical examination, he was febrile $\left(38.2^{\circ} \mathrm{C}\right)$, tachycardic (103 per minute), and hypertensive (203/126 mm Hg). Apart from an erythrocyte

published online April 15, 2020
DOI https://doi.org/

$10.1055 / \mathrm{s}-0039-3401395$

ISSN 2457-0214. rate (ESR) of 40, other routine laboratory investigations were unremarkable. A portal venous phase computed tomography (CT) study showed a wedged-shaped focal hypodensity in the right kidney. The differential diagnosis at this stage was that of focal segmental renal infarct or acute pyelonephritis. The patient was investigated for probable infective endocarditis causing the renal infarct. A transthoracic echocardiogram did not show evidence of vegetation or thrombus. Apart from the fever and a raised ESR, he did not fulfill the criteria for infective endocarditis. Pheochromocytoma work-up and a vasculitis screen were also negative. Magnetic resonance imaging (MRI) of the brain was normal. He was treated for acute pyelonephritis and discharged home with oral antibiotics, with plans for repeat imaging at 1 month.

The repeat CT showed an irregularity of the celiac trunk and stenosis of the proximal splenic artery. In addition, there was a $2.3 \times 1.2 \times 1.5 \mathrm{~cm}$ fusiform SAP. The neck of the pseudoaneurysm measured $2 \mathrm{~mm}$. Also noted was a nonocclusive focal dissection of the left renal artery ( - Fig. 1). On review of the initial portal venous phase CT performed 4 weeks earlier, the pseudoaneurysm and abnormal visceral arteries

\section{License terms \\ ()(1) $\odot \circledast$}


were noted to be present and remained unchanged. The right segmental renal infarct had remained stable, and the right renal artery appeared unremarkable. The patient was admitted for further work-up of the abnormal CT findings. Inflammatory markers were improving, and repeat blood cultures were negative. Vasculitis and autoimmune markers including antimyeloperoxidase, anti-PR3 antineutrophil cytoplasmic antibodies, enzyme immunoassay profile, lupus anticoagulant, and anticardiolipin antibody were negative. The only positive result was a weakly positive antinuclear antibody with a homogenous pattern. A CT aortogram of the thorax

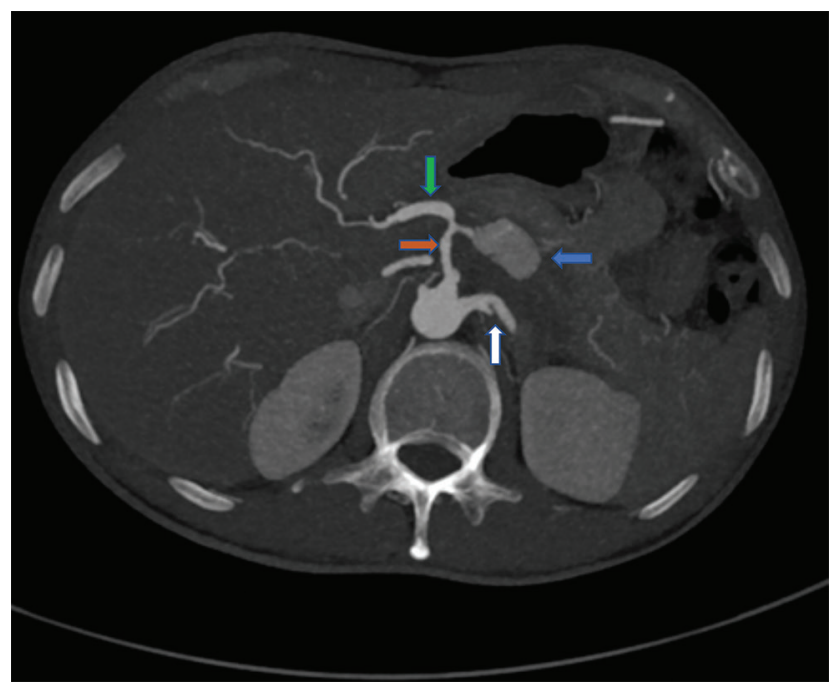

Fig. 1 Maximum intensity projection of abdominal CT (computed tomography) showing the splenic artery pseudoaneurysm (blue arrow), left renal artery dissection (white arrow), irregularity of the celiac axis (orange arrow), and ectasia of the common hepatic artery (green arrow). and abdomen showed no evidence of dissection of aneurysm formation elsewhere. Magnetic resonance (MR) angiogram of the intracranial vessels did not show any flow-limiting lesions. The overall clinical assessment did not reveal systemic vasculitis based on history and physical examination nor there were features of hypermobility or evidence of connective tissue disorders. Given the clinical, radiological, and biochemical findings, the differential diagnoses for this nonatherosclerotic, noninflammatory disease include segmental arterial mediolysis (SAM), fibromuscular dysplasia, and noninflammatory connective tissue diseases.

As the patient and his wife were planning to commence treatment for unexplained infertility, they were not keen on major surgical intervention. Therefore, following discussions between our vascular surgery and interventional radiology teams, it was agreed that urgent TAE of the aneurysm should be considered in view of the size of the pseudoaneurysm. Celiac trunk angiography through a transfemoral route showed focal stenosis in the proximal splenic artery, precluding microcatheter access into the aneurysm sac (-Fig. 2). In addition, B-mode ultrasound demonstrated an intimal dissection flap at the origin of the pseudoaneurysm ( - Fig. 3 ). The procedure was therefore aborted. Following further multidisciplinary discussions, a second attempt to treat the aneurysm through a percutaneous transgastric-transpancreatic route was suggested.

Prior to commencing the procedure, a broad-spectrum antibiotic (Cefazolin $1 \mathrm{~g}$ ) was administered intravenously as prophylaxis. The procedure was performed under local anesthesia. Using ultrasound guidance, a 22-gauge Chiba needle was introduced percutaneously using a transgastric-transpancreatic approach. A total of $1 \mathrm{~mL}(1,000 \mathrm{U})$ of thrombin (Thrombin-JMI, Pfizer Laboratories) was incrementally injected into the aneurysm sac using color Doppler
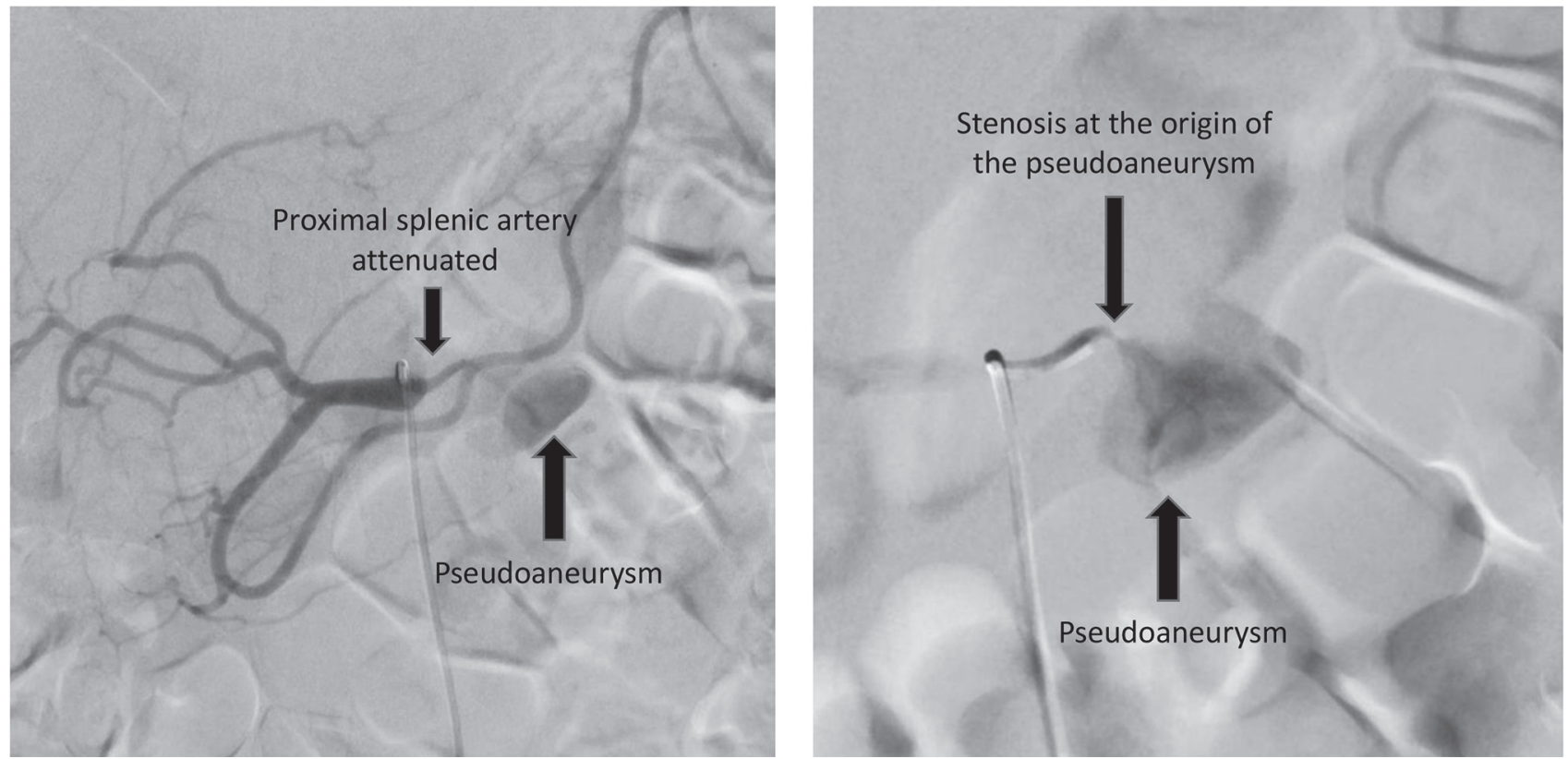

Fig. 2 Celiac trunk angiogram showing attenuated proximal splenic artery (short black arrow) with a focal high-grade stenosis at the origin of the pseudoaneurysm. 


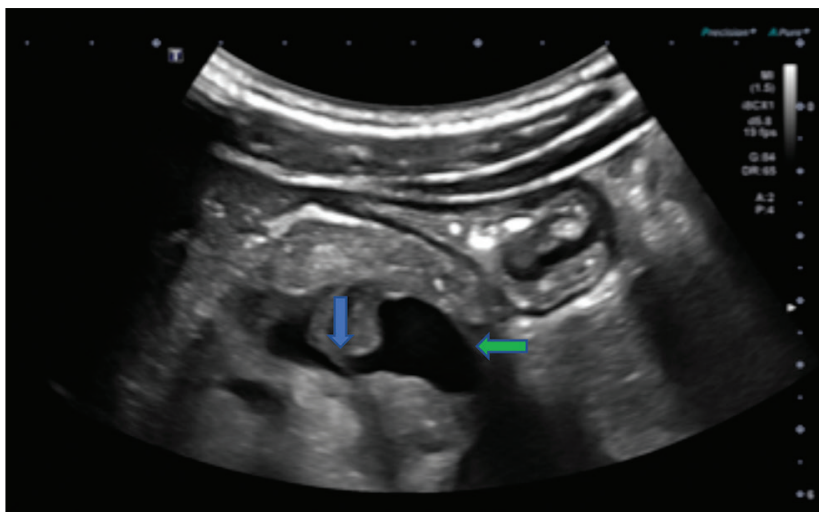

Fig. 3 B-mode ultrasound in the axial plane showing the intimal dissection flap (blue arrow) causing stenosis at the origin of the pseudoaneurysm (green arrow).

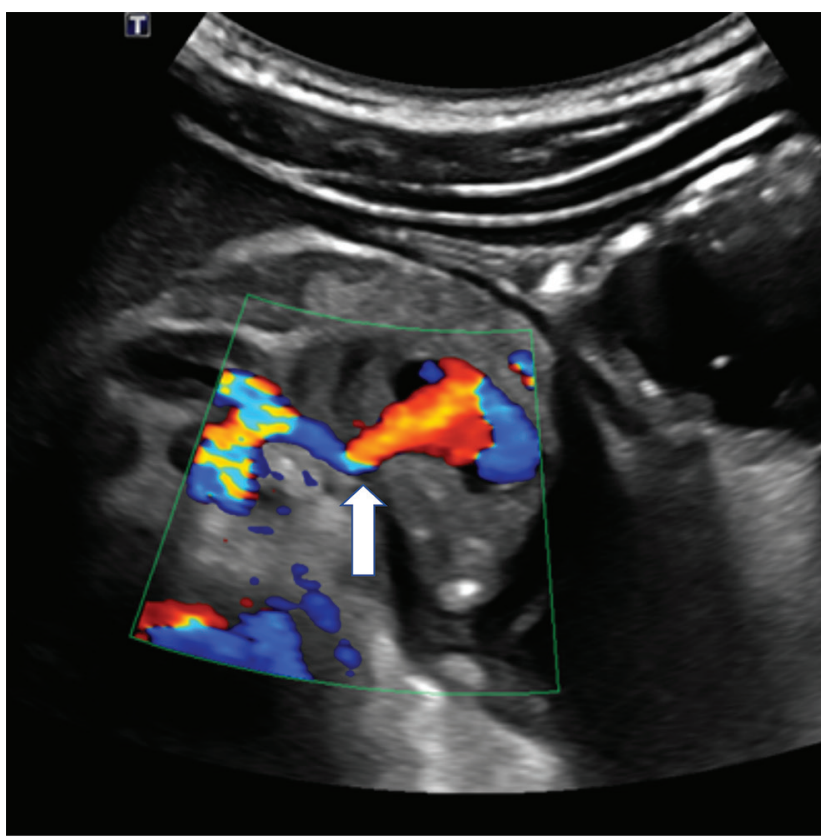

Fig. 4 Color Doppler ultrasound study demonstrating the yin-yang sign of the splenic artery pseudoaneurysm with a focal stenosis at the origin of the pseudoaneurysm.

ultrasound imaging until cessation of flow was observed within the pseudoaneurysm sac ( - Fig. 4). An immediate posttreatment arterial phase CT study confirmed thrombosis of the pseudoaneurysm ( $\mathbf{- F i g .} \mathbf{5 a}, \mathbf{b})$.

Postprocedurally, there was a transient elevation of serum amylase from 76 to 159 on day 2, which subsequently decreased to 130 on day 3 postprocedure. The patient went on to make a good recovery and was discharged home on day 3. The patient has been followed up in the outpatient clinic and remains asymptomatic. A follow-up CT aortogram showed thrombosis of the splenic artery and the pseudoaneurysm sac. There was no evidence of splenic infarction. There were, however, new luminal irregularities and narrowing of the proper and left hepatic arteries.

\section{Discussion}

Segmental arterial mediolysis is a rare nonatherosclerotic, noninflammatory cause of SAPs. ${ }^{1}$ First reported in 1976 by Slavin and Gonzalez-Vitale, ${ }^{2}$ the disease affects all age groups and has a slight male preponderance. The etiology of SAM is still unclear. The primary pathological finding appears to be lysis of the medial layer of the muscular arterial wall caused by disruption of the smooth muscle cell membrane. The definitive diagnosis of SAM requires histological confirmation, which very often is not possible. The disease affects all parts of the body, with the most common site being the celiac trunk and its branches, specifically the splenic artery. ${ }^{3}$ Typical angiographic findings of SAM include arterial dissections, stenosis, occlusions, and aneurysms. ${ }^{2}$ The diagnosis of SAM was considered based on clinical, imaging, and biochemical findings in this case.

It is important to emphasize that TAE remains the standard-of-care first-line management of visceral artery aneurysms or pseudoaneurysms. TAE using a variety of embolic agents such as microcoils is effective and remains the firstline treatment for splenic artery aneurysms. The challenge arises in cases in which endoluminal treatment is technically hindered by significant stenosis. In our case, TAE was unsuccessful due to the proximal stenosis and possible dissection flap. The etiology of the stenotic plaque is likely because of reparative granulation tissue formation due to the underlying vascular pathology ( - Fig. 3). As was observed in our case, the proximal stenosis made catheterization of the neck of the aneurysm technically challenging, and deployment of a proximal coil would potentially have risked coil migration with subsequent nontarget embolization.

Percutaneous direct thrombin injection is widely accepted to be a simple, safe, and effective treatment for iatrogenic postcatheterization femoral artery pseudoaneurysms. ${ }^{4-6}$ Thrombin injection also has a role in treating pancreatic pseudoaneurysms that develop following an episode of acute pancreatitis. ${ }^{7}$ Thrombin acts by converting fibrinogen into fibrin, leading to thrombus formation. In comparison with endovascular techniques, percutaneous thrombin injection has the advantage of not being dependent on vascular anatomy. Complication rates are low and may include thromboembolic events from thrombin escaping into the arterial system and, rarely, allergic reactions with the use of bovine thrombin.

Intuitively, one would assume that ultrasound-guided percutaneous needle entry into the pancreas can cause acute pancreatitis. However, a study on ultrasound-guided percutaneous fine needle aspiration of solid pancreatic neoplasms in more than 2,000 cases showed a complication rate of only $0.8 \%{ }^{8}$ There are also reports showing the feasibility of using endoscopic ultrasound (EUS) guided thrombin injection for the treatment of visceral artery pseudoaneurysms. ${ }^{9}$ Similar to our case, EUS-guided thrombin injection involves transgression of the gastrointestinal wall, contributing to a small potential risk of introducing infection. 

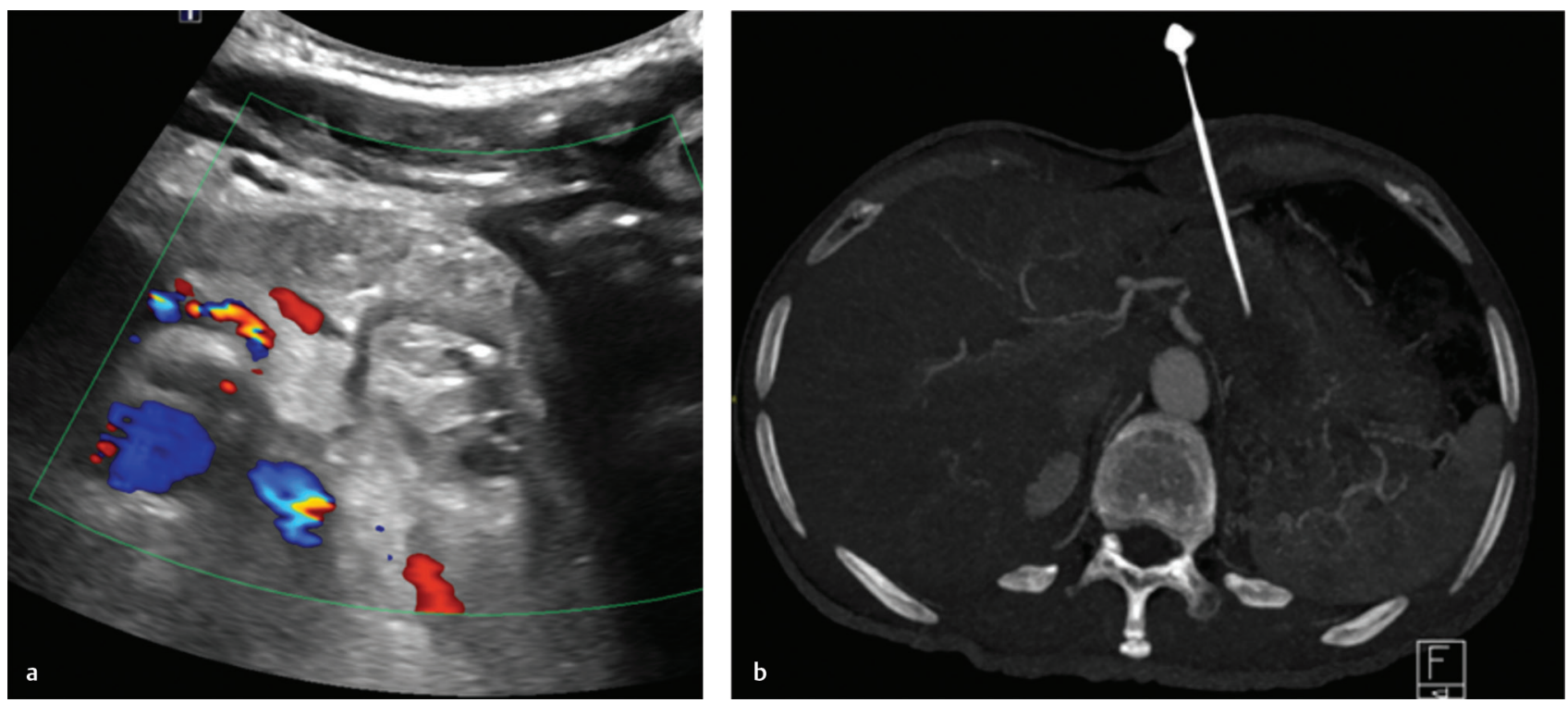

Fig. 5 (a) Doppler ultrasound image showing postthrombin injection and (b) postprocedure CT (computed tomography) showing needle within the thrombosed aneurysm sac.

Long-term follow up is necessary for SAM due to its spatiotemporal characteristics and potential for progression. ${ }^{10} \mathrm{We}$ acknowledge the short duration of follow-up in our patient, in whom the aneurysm remains thrombosed. Nevertheless, there is no consensus regarding follow-up imaging for SAM. In view of the patient's young age, a CT aortogram 1 year following treatment was considered a pragmatic interval for follow-up imaging.

\section{Conclusion}

Direct percutaneous thrombin injection into a SAP through a transgastric-transpancreatic route can be used as an alternative approach when the preferred first-line endovascular option is unsuccessful. In the case presented, SAM remains a tentative diagnosis in the absence of histopathological confirmation.

\section{Conflicts of Interest}

None.

\section{References}

1 Noh SY, Shin JH, Yoon H-K, Ko G-Y, Sung K-B. Segmental arterial mediolysis: literature review focused on radiologic findings and management. Gastrointest Interv 2016;5(1):22-26

2 Slavin RE. Segmental arterial mediolysis: a clinical-pathologic review, its role in fibromuscular dysplasia and description and differential diagnosis of the masquerader-muscular artery cystic necrosis. World J Cardiovasc Dis 2013;03(1):64-81
3 Pillai AK, Iqbal SI, Liu RW, Rachamreddy N, Kalva SP. Segmental arterial mediolysis. Cardiovasc Intervent Radiol 2014;37(3):604-6124 Kang SS, Labropoulos N, Mansour MA, et al. Expanded indications for ultrasound-guided thrombin injection of pseudoaneurysms. J Vasc Surg 2000;31(2):289-298

5 Elford J, Burrell C, Freeman S, Roobottom C. Human thrombin injection for the percutaneous treatment of iatrogenic pseudoaneurysms. Cardiovasc Intervent Radiol 2002;25(2):115-118

6 Saad NE, Saad WE, Davies MG, Waldman DL, Fultz PJ, Rubens DJ. Pseudoaneurysms and the role of minimally invasive techniques in their management. Radiographics 2005;25 (1, Suppl 1):S173-S189

7 Chauhan U, Puri SK, Jain N, et al. Percutaneous thrombin injection under sonographic guidance for exclusion of noncatheterizable post-pancreatitis pseudoaneurysm of the superior mesenteric artery: a minimally invasive and expeditious treatment option. J Med Ultrason (2001) 2016;43(2):295-299

8 D'Onofrio M, De Robertis R, Barbi E, et al. Ultrasound-guided percutaneous fine-needle aspiration of solid pancreatic neoplasms: 10-year experience with more than 2,000 cases and a review of the literature. Eur Radiol 2016;26(6): 1801-1807

9 Gamanagatti S, Thingujam U, Garg P, Nongthombam S, Dash NR. Endoscopic ultrasound guided thrombin injection of angiographically occult pancreatitis associated visceral artery pseudoaneurysms: case series. World J Gastrointest Endosc 2015;7(13):1107-1113

10 Hashimoto T, Deguchi J, Endo H, Miyata T. Successful treatment tailored to each splanchnic arterial lesion due to segmental arterial mediolysis (SAM): report of a case. J Vasc Surg 2008;48(5):1338-1341 